\title{
Calculated index of the compacting of sea ice cover from satellite images
}

\author{
A. I. Aleksanin, M. G. Aleksanina, A. Yu. Karnatsky \\ Institute of Automation and Control Processes FEB RAS, Vladivostok, 690041, Russia \\ E-mail: aleks@iacp.dvo.ru
}

\begin{abstract}
This paper describes a new method of automatic calculation of local indices of the compacting and divergence of sea ice cover. The proposed approach is based on the calculation of ice drift velocities. The local index of the compacting and divergence of sea ice cover is considered as the rate of change in the distance between individual elements of the sea ice cover. The local index of compacting and divergence is determined by two parameters: the scalar value of compacting/divergence and the direction of the axis of compacting/divergence. This approach allows the estimation of the accuracy and statistical significance of the calculated parameters. It was shown that the proposed approach results correspond to visually observed parameters of compacting and divergence.
\end{abstract}

\section{Introduction}

Ice compacting that occurs in freezing seas due to uneven ice drift is most dangerous for shipping [1]. According to the guidance on methods for measuring the characteristics of ice cover dynamics [2], the "pure" dynamics of the sea ice cover is understood as variability of the ice element arrangement in the time. The measure of ice compacting is usually determined visually by the ice field shape [3]. To formalize the ice compacting definition, the speed of closing edges of a lead channel behind an icebreaker is often used. In this case, there is a definite relationship between the degree of compacting and the speed of the channel edge closure [1].

The most suitable data to monitor the sea ice cover are the operational data from meteorological satellites. Two approaches are applied to determine zones of the ice field compacting using the satellite images. The first approach considers occurrence of compacting as a thickening of cracks based on the adapted methods of lineament analysis that was previously used to study land patterns [4]. It is pointed out that the type of orientation of ice breaks relative to the general icebreaker course is one of the most important ice characteristics for navigation in the Arctic basin [1,5-7]. The second approach that is more generally accepted is to search for the zones of compacting. This approach relies on the calculation of the divergence and shift of the vector field of ice drift velocities [8, 9]. The synthetic aperture radar images with high spatial resolution are mainly used. The coverage area provided by the radar is small and does not provide regular information for an arbitrary sea point. The compacting directions are not calculated, although they are an important characteristic for the ice escort of ships [5]. There are no specific methods to determine the direction of the ice field compacting using the satellite information. Despite the available technologies to process the satellite data, interactive interpretation methods still prevail. 
In the present paper, a new method is proposed to estimate the direction and magnitude of the ice compacting using the satellite images. The essence of the proposed approach is to consider the compacting/divergence as the rate of change in time of the distance between the observed medium elements and to identify the direction of the greatest rate.

\section{Calculation of ice compacting}

\subsection{Calculation of the local indices of compacting/divergence}

At the first step, ice drift rates are constructed. To calculate the ice drift velocity, the method developed in [10] is used. It is an analogue of the method of maximum cross-correlation (MCC) [11] and allows to reduce the drawbacks of MCC. In a grid of the given size of $L x \times L y$ pixels, the velocities of the ice displacement are calculated using two images got with the time interval from several hours to days. The second step is to estimate the indices of compacting and divergence using the calculated velocities of the sea ice cover displacements.

The essence of the proposed approach is that the compacting is considered as reduction $(-)$ of the distance between two moving points on a pair of images, and the divergence, on the contrary, is considered as increase $(+)$ of this distance. The pair of the first image points forms a vector $\mathbf{R}^{k}$ that begins in the grid node $X_{i j}$, for which the compacting/divergence parameters are calculated (Figure 1a). The grid node positions to calculate the parameters coincide with the grid node positions to calculate the ice drift, but these nodes are several times less frequent.

Let's define the local index of compacting or divergence $C$ as a change in the length of the vector $\mathbf{R}^{k}$ per unit time (Figure 1a):

$$
C_{i j}^{k}=\frac{S_{1}-S_{0}}{\Delta t}
$$

where $\Delta t$ is the time interval between the satellite images, $S_{0}$ is the initial distance between points, $S_{1}$ is the finite distance.
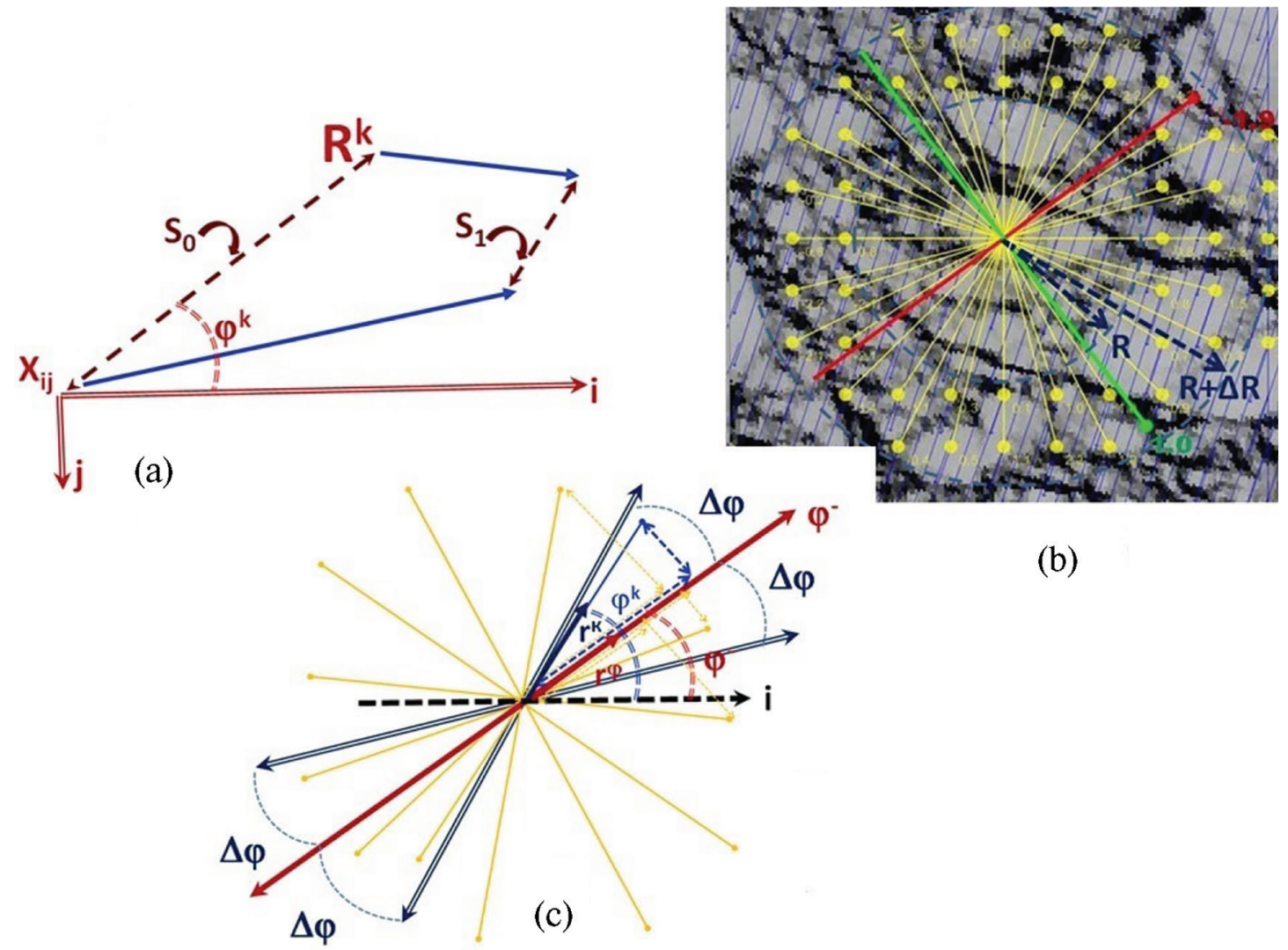

(b)

Figure 1. Calculation scheme (a) local compacting index $C_{i j}^{j}$; (b) the "rose" of local compacting indices in the torus $\Omega$ with radii $R, R+\Delta R$, red colour indicate the direction of compacting, and green colour indicate direction of divergence; (c) the angle $\varphi^{-}$indicate the compacting direction. 
The local compacting indices are calculated for different directions $\mathbf{R}^{k}$ that are characterized by the angle $\varphi^{k}$ (Figure 1a). The compacting index $C^{-}$is calculated in the vicinity of the studied point $X_{i j}$ in the torus $\Omega$ with radii $R_{1}$ and $R_{2}=R_{1}+\Delta R$ based on the local indices $\left\{C_{i j}^{k}\right\}: k \in \Omega_{R, \Delta R}$ (Figure lib). The compacting direction is characterized by the angle $\varphi^{-}$(Figure 1c). The local compacting indices along this direction should reach the minimum values. The compacting index is characterized by the calculation accuracy $\alpha$, and the compacting direction is characterized by the angle calculation accuracy $\Delta \varphi$.

\subsection{Preliminary rejection of the values $C^{k}$ for the given point $X_{i j}$}

The local indices pass three rejection levels. At the first step, the "surges" are rejected. Let we get $N_{0}$ values of $\left\{C^{k}\right\}$ for the selected point under the specific cloud conditions. To reject the values using the criterion $\left|C^{k}\right|>\Delta S_{t}^{*}$, the threshold is chosen based on the physical sense as the maximum possible rate of change of the distance between the displacement points. It is remained $N_{1}$ values of $\left\{C^{k}\right\}$. At the second step of the rejection, we make statistical estimates. We estimate the average $M\left(C^{k}\right)$ and root-mean-square deviation $\sigma\left(C^{k}\right)$, and then reject those values $\left\{C^{k}\right\}$, for which $\left|C^{k}-M\left(C^{k}\right)\right|>2 \sigma\left(C^{k}\right)$. After the second rejection procedure, it is remained $N_{2}$ values $\left\{C^{k}\right\}$. In the third step, we estimate the number of the remained local estimates for the values $\left\{C^{k}\right\}$. If it is remained less than three quarters of the maximum possible number of values, we do not detect the compacting/ divergence in the point $X_{i j}$

\subsection{Estimation of the direction of the compacting/divergence axis}

The vectors $\mathbf{R}^{k}$ normalized by their length with weights that are the local compacting values are projected to the given direction characterized by the angle $\varphi^{-}$(Figure 1c) and the average value of the projections is calculated by the formulas:

$$
C^{\varphi}=\frac{1}{N_{2} \sum C^{k}} r^{\varphi} r^{k}
$$

where the vector $\mathbf{r}^{\varphi}=(\cos \varphi, \sin \varphi)$ for $\varphi^{k} \in(-\pi / 2,+\pi / 2)$ and $-\mathbf{r}^{\varphi}$ for the remaining angle range, $\mathbf{r}^{k}=\left(\cos \varphi^{k}, \sin \varphi^{k}\right)$. The ice compacting direction $\varphi^{-}$at the given point is determined by the optimal choice of $\varphi$ that ensures the minimum value of $C^{\varphi}$. The search for the maximum of $C^{\varphi}$ gives an estimate of the divergence direction angle $\varphi^{+}$. The direction is searched through the angles with the specified step.

\subsection{Estimation of the compacting/divergence value}

To obtain the compacting value, we assume that the ice is compacted along the specific direction and the compacting value is the same for the entire neighbourhood of the calculation point. Then, in the absence of errors in calculating the displacement velocities, the compacting value can be obtained from the local compacting:

$$
C^{-}=\frac{C^{k}}{\cos \left(\varphi^{-}-\varphi^{k}\right)}
$$

Due to presence of noise, an average estimate of $C^{-}$should be used. But when the value of $\left|\varphi-\left(\varphi^{k}\right)\right|$ comes close to $\pi / 2$, the errors are sharply increased due to the tendency of the cosine to zero. Therefore, the compacting value is calculated not for all values of the local compacting, but only for those that lie in a certain range of angles $\Delta \varphi$ (Figure 1c). The angle range is selected to optimize the accuracy estimation for the calculation of $C^{-}$. $C^{-}$is calculated by the formula:

$$
C^{-}=\frac{1}{n} \cdot \frac{\sum C^{k}}{\cos \left(\varphi^{-}-\varphi^{k}\right)},
$$

where $n$ is the number of local compacting vectors lying in the angle range. In this case, the local vector number must be more than some reasonable threshold $n^{*}$, that makes it possible to estimate the calculation accuracy by statistical methods. The angle range is searched by exhaustive search. 


\subsection{Estimation of the calculation accuracy}

Practice shows that the distribution of the sum terms in expression (4) can be regarded as a distribution close to the normal (Gaussian) distribution. To calculate a confidential interval where the true value of the average will fall with a probability of $P_{r b}=95 \%$, and taking into account the limited number of measurements, it is necessary to use the Student's distribution. In our case, to estimate the compression value $C^{-}$, we obtain the confidential interval or the absolute error as:

$$
\Delta C^{-}=\sigma_{C}^{-} \frac{t_{f, 0.95}}{\sqrt{n}}
$$

where $t_{f, 0.95}$ is the Student's coefficient. The calculation accuracy is shown by the relative error value:

$$
\frac{\Delta C^{-}}{C^{+}}=\frac{\left(\sigma_{C}^{-} t_{f, 0.95}\right) / \sqrt{n}}{C^{-}}=\alpha,
$$

We will use only those compacting estimates, which values provide the given accuracy. It is logical to define the calculation accuracy estimation for the compacting direction $\Delta \varphi^{-}$as a deviation that causes degradation of the relative calculation accuracy up to an allowable value $\alpha$. Therefore, the deviation of the calculated compacting direction from the true direction to the angle $\Delta \varphi^{-}$, giving the error $\alpha C^{-}$, reduces to the equation:

$$
C^{-}-C^{-} \cdot \cos \Delta \varphi^{-}=\alpha C^{-} .
$$

Hence, if the accuracy of calculating the compacting value $\alpha$ is known, an estimate of the compacting direction accuracy $\Delta \varphi^{-}$can be obtained:

$$
\Delta \varphi^{-}=\arccos (1-\alpha)
$$

Similarly, the divergence parameters are calculated too.

The compacting value will depend on the size of the torus with a characteristic radius $R$, which we take as the value $\left(R_{1}+R_{2}\right) / 2$. To estimate the compacting value behaviour as a function of the torus size, we will use the relative compacting value $C_{n}^{-}$that which is calculated using the same formulas, but with the local indices $C_{n i j}^{k}=\left(S_{1}-S_{0}\right) \cdot \Delta t^{-1} \cdot\left(S_{0}\right)^{-1}$ that are normalized by the distance. It should be noted that the relative compacting dimension corresponds to the divergence dimension.

\section{Testing the proposed method}

A pair of the images fragments of the Sea of Okhotsk in Mercator projection with calibration of values and spatial resolution of $250 \mathrm{~m}$ per pixel for the data of the MODIS/Aqua\&Terra radiometer was selected for testing. The time interval is taken within 24 hours. The ice compacting can be observed visually by the crack width $(\sim 500 \mathrm{~m})$ of the fractured ice floe in the image centre on the left and by the same but compacted crack, in figure 2 on the right.

For the images (Figure 2), the ice compacting parameters were calculated for tori with characteristic radii of $7-22 \mathrm{~km}$ (from 30 to 110 pixels) with an allowable relative error $\alpha=0.5$. Figure 3 shows graphs of the variability of the compacting values and directions for different characteristic sizes of the calculation area (the ratio of the radii $R_{1}$ to $R_{2}$ in pixels: $30-50 ; 50-70 ; 70-90 ; 90-110 ; 110-$ $130 ; 130-150 ; 210-230 ; 290-310 ; 490-510)$ for arbitrary 13 points $X_{i j}$ near the fractured ice floe for April 5-6, 2010, according to the images of the MODIS/Aqua radiometer.

It should be noted that, for the radii $R_{1}$ and $R_{2}$ of 30-50 pixels, the compacting indices did not fall within the confidential interval of $95 \%$ with the allowable relative error $\alpha=0.5$, mainly due to insufficient data to get a statistically significant result. You can see from the graph that there are the points where stability of the relative compacting is observed at distances from $120 \mathrm{~km}$. As the radius of the torus increases after reaching a certain size, the expected decrease in the relative compacting value is observed. The compacting directions in the vicinity of the selected ice floe were quite stable and changed only slightly with increasing the torus radii. Significant variability appeared only at large radii (more than $100 \mathrm{~km}$ ). At the same time, significant variability of the parameter (in 2 or more times) is observed for many points. This indicates the uneven compacting in space, which results in dependence 
of this parameter on the characteristic size of the calculation windows. As the radius of the torus increases after reaching a certain size, the expected decrease in the relative compacting value is observed. The compacting directions in the vicinity of the selected ice floe were quite stable and changed only slightly with increasing the torus radii. Significant variability appeared only at large radii (more than $100 \mathrm{~km})$.

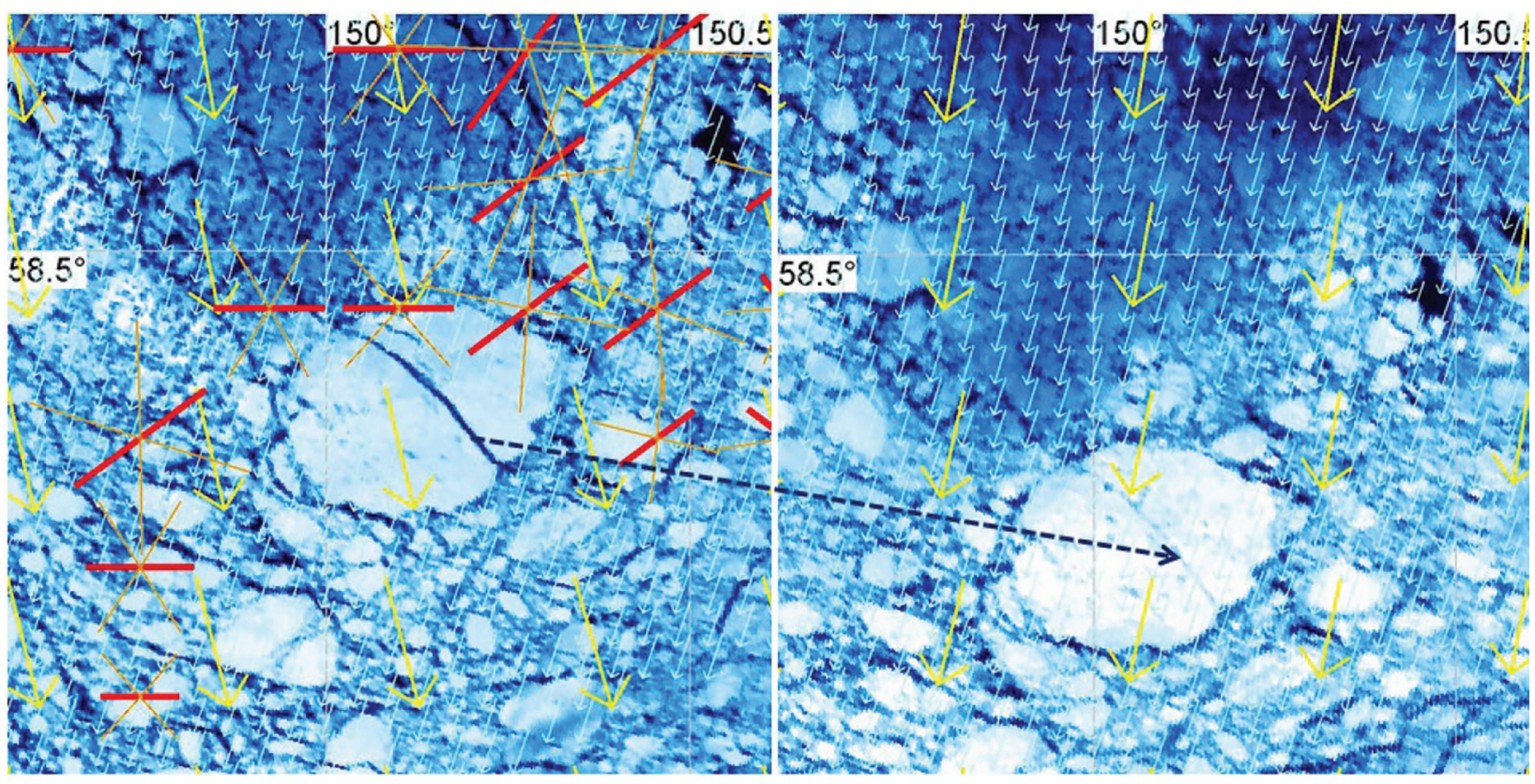

Figure 2. An example of visually observed compacting (red) of the sea ice cover on fragments of MODIS/Aqua images: on the left - for April 5, 2010, on the right - for April 6, 2010. Yellow vectors - wind speeds, blue vectors - automatically calculated ice drift velocities. Thin yellow-red lines indicate the permissible orientation of the compression axis $\Delta \varphi^{-}$.

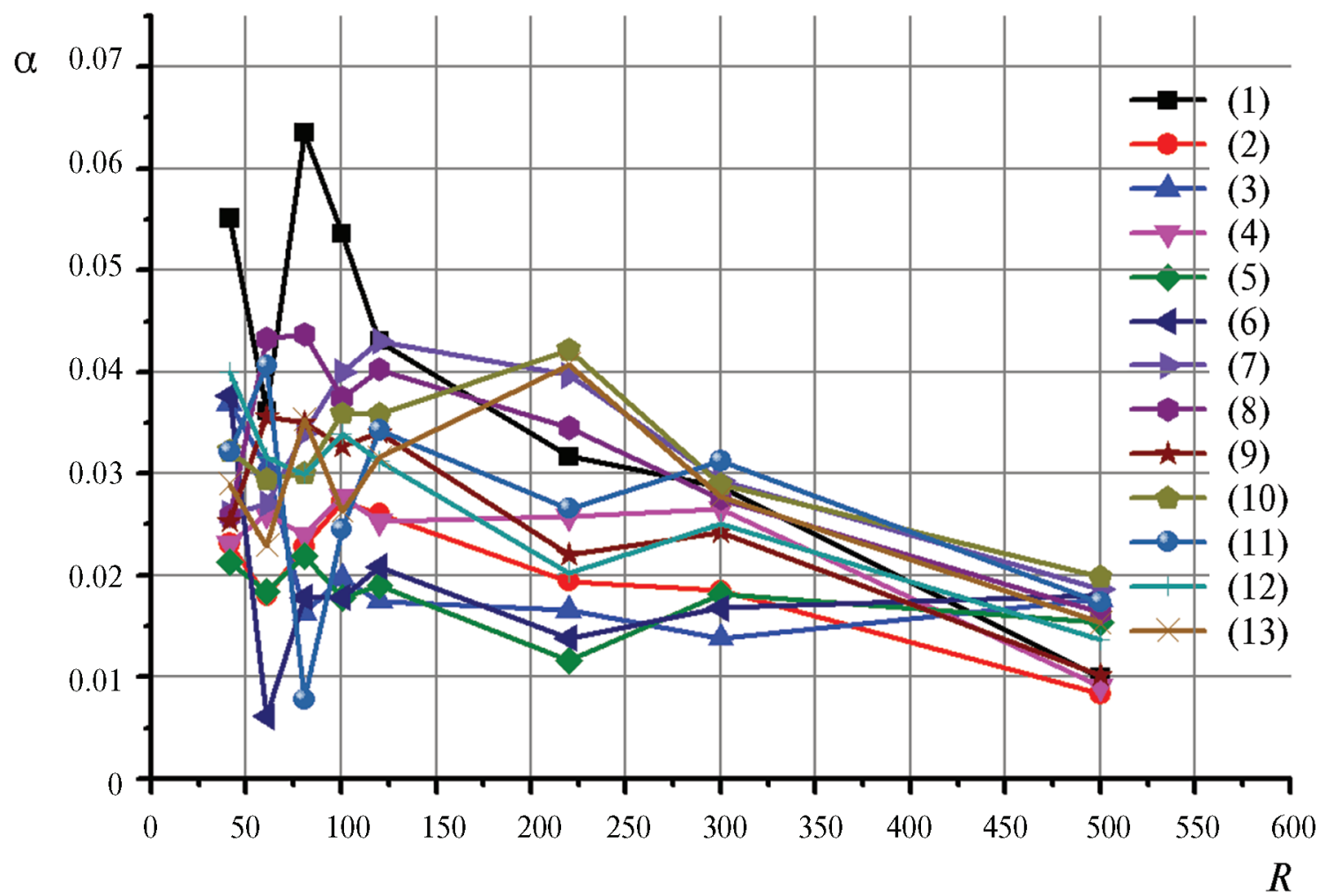

Figure 3. Variability of the compacting values $\alpha$ for different characteristic sizes of the calculation area for arbitrary 13 points $X_{i j}$ near the fractured ice floe for April 5-6, 2010 (Figure 2). 


\section{Discussion}

If we consider the compacting over a large area of the Sea of Okhotsk (Figure 4), then it can be noted that the expected regularities are observed. Two zones are observed: the northern zone where the compacting dominates and southern one where the divergence dominates. The southern zone corresponds to increase in the wind speed that can explain the divergence (stretching) of the ice field. Sometimes there are cases when the compacting and divergence present in the point simultaneously, but in the different directions that often are perpendicular to each other.

The key question of any algorithm being created is the calculation accuracy of the parameters to be estimated. Unfortunately, the compacting value calculated automatically can be compared only with visually-manual estimates obtained using the same images. The compacting force estimation by the ice cover form speaks more about the previous deformations than about the actual compacting value. The calculation of the closure speed for the channel made broken by the icebreaker is usually not practiced, since it requires taking measurements.

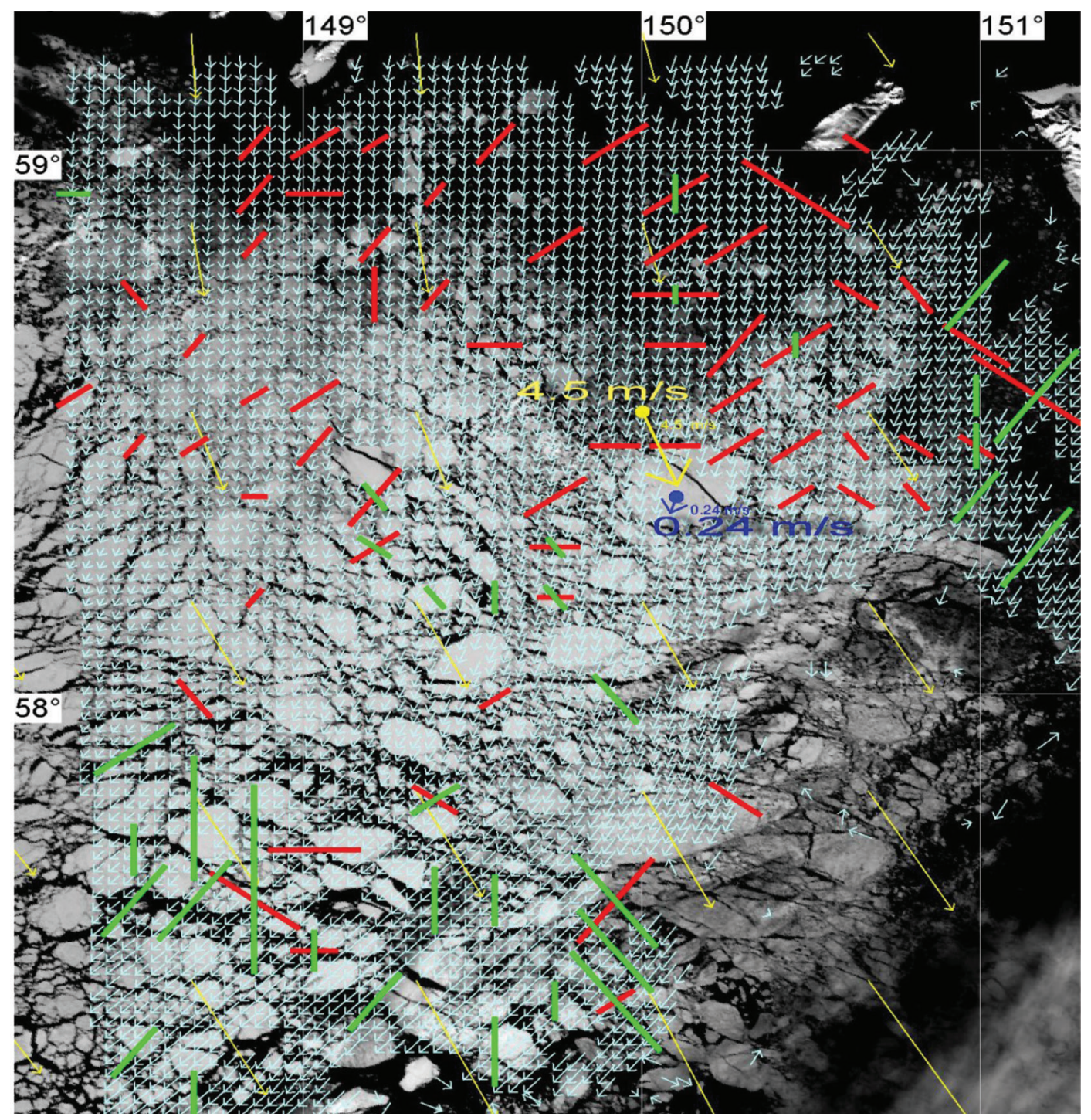

Figure 4. Result of automatic detection of the compacting (red)/divergence (green) zones based on drift vectors with a permissible relative error $\alpha=0.5$.

The compacting value to be calculated will also depend significantly on the time interval between the images. We used the daily time interval in our experiments, as it is recommended for the ice drift calculations [12], whereas the typical ice field compacting lasts several hours. Reducing the time interval leads to increase in the drift calculation error caused by the accuracy of the image spatial resolution. 


\section{Summary}

An approach is proposed to determine the zones of ice compacting and automatic calculation of the compacting value and direction. This approach is based on the analysis of the displacement velocity field of ice fields calculated by the new algorithm for the time sequence of meteorological earth satellite images. The statistically significant estimates of the relative compacting are analysed for the given calculation accuracy. The relative compacting is essentially analogous to the value of the ice field convergence in the direction providing the maximum compacting. Despite the small relative compacting values $1-5 \%$ of the distance between the traced pairs of the points), the compacting parameters are estimated confidently with a sufficiently high level of statistical significance and they can be trusted.

\section{Acknowledgements}

This work was supported by Presidium RAS, Program "Fundamental aspects of supercomputing for simulating complex practical problems".

\section{References}

[1] Appolonov E. M., Sazonov K. E., Bokatova E. A., On the probability of sticking of vessels under ice pressure, The World of Transport, 2012, Vol. 10(4(42)), pp. 4-9.

[2] Stepanyuk I.A., Smirnov V.N., Methods for measuring the characteristics of ice cover dynamics, Saint Petersburg: Gidrometeoizdat, 2001, 136 p.

[3] Slivaev B. G., Preparation of the vessel for navigation in ice, Vladivostok: IPK MGU im. adm. G. I. Nevel'skogo, 2017, $67 \mathrm{p}$.

[4] Pyatkin V.P., Salov G. I., Statistical Approach to the Problem of Some Structures Detection in Aerospace Imagery, Science-intensive technologies, 2002, Vol. 3, No. 3, pp. 52-58.

[5] Babich N. G., Selecting navigation routes and accessing efficiency of ice navigation data application, Earth from Space, 2011, No. 10, pp. 28-33.

[6] Frolov S. V., Orientation of the leads and cracks in the ice cover relatively to direction of the ship movement is the most important characteristic of ice navigation in the arctic basin, Arctic and Antarctic Research, 2013, No. (97), pp. 35-45.

[7] Gol'dshtein R.V., Osipenko N. M., Fracture mechanics and the problems of development of the Arctic, Arctic: Ecology and Economy, 2015, Vol. 4(20), pp. 14-27.

[8] Yu J., Yang Y., Liu A., Zhao Y., Analysis of sea ice motion and deformation in the marginal ice zone of the Bering Sea using SAR data, Intern. J. Remote Sensing, 2009, Vol. 30(140), pp. 3603-3611.

[9] Bouillon S., Rampal P., On producing sea ice deformation dataset from SAR-derived sea ice motion, The Cryosphere Discuss, 2014, Vol. 8, pp. 5105-5135.

[10] Aleksanin A. I., Aleksanina M.G., Karnatskii A. Yu., Automatic computation of sea surface velocities on a sequence of satellite images, Sovremennye problemy distantsionnogo zondirovaniya Zemli iz kosmosa, 2013, Vol. 10(2), pp. 131-142.

[11] Emery W. J., Thomas A. C., Collins M. J., Crawford W. R., Mackas D. L., An objective method for computing advective surface velocities from sequential infrared satellite images, J. Geophysical Research, 1986, Vol. 91(C11), pp. 12865-12878.

[12] Lavergne T., Eastwood S., Teffah Z., Schyberg H., Breivik L.-A., Sea ice motion from low-resolution satellite sensors: An alternative method and its validation in the Arctic, J. Geophysical Research, 2010, Vol. 115(C10), C10032, 14 p. 